\title{
CONFORMAL MAPPINGS OF CIRCULAR DOMAINS ON FINITELY-CONNECTED NON-SMIRNOV TYPE DOMAINS
}

\author{
F.G. AVKHADIEV, P.L. SHABALIN
}

\begin{abstract}
We consider a canonical factorization and integral representation for the derivatives of the conformal mappings of circular domains on finitely-connected non-Smirnov type domains. By means of the functions in the Zygmund class, we obtain the conditions for the global univalence. Earlier similar results were obtained by a series of authors just for simply-connected domains.
\end{abstract}

Keywords: Non-Smirnov type domain, Zygmun condition, Schwarz operator.

Mathematics Subject Classification: 30C45, 30C55, 30C62, 31A20

\section{INTRODUCTION}

We consider conformal mappings $f: D \rightarrow \Omega$ of the unit circle $D=\{z \in \mathbb{C}:|z|<1\}$ on Jordan domains $\Omega$ with rectifiable boundaries. It is know that [1] that $f^{\prime}$ belongs to the Hardy class $H^{1}(D)$ by the Riesz theorem and the Smirnov canonical representation holds. This representation is determined by the formulae $f^{\prime}=e^{i \gamma}\left(f^{\prime}\right)^{e x t}\left(f^{\prime}\right)^{i n t}$,

$$
\ln \left(f^{\prime}\right)^{e x t}(z)=\frac{1}{2 \pi} \int_{-\pi}^{\pi} \frac{1+z e^{-i \theta}}{1-z e^{-i \theta}} \ln \left|f^{\prime}\left(e^{i \theta}\right)\right| d \theta, \quad \ln \left(f^{\prime}\right)^{i n t}(z)=\frac{1}{2 \pi} \int_{-\pi}^{\pi} \frac{1+z e^{-i \theta}}{1-z e^{-i \theta}} d \mu(\theta),
$$

where $\gamma=$ const $\in \mathbb{R}, \mu$ is a continuous increasing function, whose derivative is zero almost everywhere. In 1928, V.I. Smirnov [2] considered first the class of the domains $\Omega$ characterized by the absence of the singular factor, that is, by the identity $\left(f^{\prime}\right)^{i n t}(z) \equiv 1$. M.V. Keldysh and M.A. Lavrentiev [3] constructed geometrically an example of a domain $\Omega$, for which $\left(f^{\prime}\right)^{\text {ext }}(z) \equiv$ 1 and in this way they proved the existence of non-Smirnov domains. A more general analytic approach for constructing non-Smirnov domains was proposed by Duren, Shapiro and Shields in 1966 [4] by applying a series of interesting facts from various fields of the theory of functions. In particular, they employed the functions obeying the Zygmund conditions [5]

$$
|\nu(\theta+\tau)-2 \nu(\theta)+\nu(\theta-\tau)|=O(\tau)
$$

to characterize the function

$$
\nu(\theta)=\mu(\theta)+\int_{0}^{\theta} \ln \left|f^{\prime}\left(e^{i t}\right)\right| d t
$$

and Ahlfors-Weill condition [6]

$$
\sup _{z \in D}\left(1-|z|^{2}\right)^{2}\left|\left(f^{\prime \prime}(z) / f^{\prime}(z)\right)^{\prime}-(1 / 2)\left(f^{\prime \prime}(z) / f^{\prime}(z)\right)^{2}\right|<2
$$

F.G. Avkhadiev, P.L. Shabalin, Conformal mappings of Circular domains on finitelyCONNECTED NON-SMIRNOV TYPE DOMAINS.

(c) Avkhadiev F.G., Shabalin P.L. 2017.

The work is supported by RFBR (project no. 14-01-00371-a).

Submitted July 25, 2016. 
ensuring the global univalence for the conformal mapping $f: D \rightarrow \mathbb{C}$ and the quasi-conformal continuability of the mapping $f$ on the entire plane. The developing of the results in [4] with applications to the theory of general and applied inverse boundary value problems was given in works [7] and [8] by the authors.

As N.G. Makarov noted in 1989 [9], the question on a complete characterization of Smirnov domains is still open. There is still no general criterium, while in this direction there is a series of interesting results. Among them we can distinguish the solution of a complicated problem obtained by P.W. Jones and S.K. Smirnov in 1999 [10]: the non-Smirnov property of a domain $\Omega$ does not imply the non-Smirnov property for its complement $\Omega^{-}:=\overline{\mathbb{C}} \backslash \bar{\Omega}$. We note that in [10] we employ essentially the results of the works [11] and [12] on local characterization of the boundary of a domain by means of harmonic measures. The further development of these results can be found in the paper by V.N. Dubinin [13].

There are well-known natural generalizations of Hardy classes and Smirnov factorization theorems for finitely-connected domains. The history of the problems, original definitions and the results of the factorization and integral representations for the multi-connected domains are described in the papers by G.Ts. Tumarkin and S.Ya. Khavinson [14], [15], G.Ts. Tumarkin [16] and D. Khavinson [17].

The aim of the present work is to generalize the results by Duren, Shapiro and Shields [4] for finitely-connected domains and extension of our results from [7] and [8] for two-connected domains. We also mention that the passage from simpy-connected domains to finitely-connected domains makes no problems if we discuss the local properties of boundary curves with no explicit estimates. But we shall be interesting in criterions for global univalence by boundary data and we have to deal with sophisticated integral representations and related estimates. And moreover, we need appropriate generalization of the aforementioned Ahlfors-Weill condition (1.2) for the global univalence. To obtain the conditions for the univalence, we shall employ essentially the methods and the results of works [18]-[23].

\section{PRELIMINARIES}

We introduce some notations and definitions. Let $D_{n}$ be a circular (that is, each connected boundary component is a circle) $n$-connected domain with non-degenerate boundary components $\gamma_{k}=\left\{z:\left|z-a_{k}\right|=R_{k}\right\}, k=\overline{1, n}$, and $a_{n}=0, R_{n}=1$ and the circle $\gamma_{n}=\{z:|z|=1\}$ envelops the others.

Definition 1. (see [14]). The Hardy class $H_{1}\left(D_{n}\right)$ is the set of holomorphic in $D_{n}$ functions $g$ such that given $g$ and $\gamma_{k}, k=\overline{1, n}$, there exists a sequence of circles $\gamma_{k}^{j} \subset D_{n}, j=1,2, \ldots$, satisfying the conditions $\gamma_{k}^{j} \rightarrow \gamma_{k}$ and

$$
\sup _{j} \int_{\gamma_{k}^{j}}|g(z) d z|<\infty .
$$

Let $f$ make a univalent mapping of $D_{n}$ onto some domain $\Omega_{n}$. We assume that the function $f$ is holomorphic in $D_{n}$ and can be continuously continued to the boundary $\partial D_{n}=\bigcup_{k=1}^{n} \gamma_{k}$ and this mapping is continued to a homeomorphism of the boundaries. In the case $n>1$ there exist two equivalent definitions of Smirnov class; the equivalence was proved in [14].

Definition 2. [14 A domain $\Omega_{n}$ with a Jordan rectifiable boundary is called a domain of Smirnov class if one of the following conditions is satisfied:

a) the function $\ln f^{\prime}$ is represented by the Schwarz operator applied to the limiting values $\ln f^{\prime}\left(\zeta_{k}\right), \zeta_{k}=a_{k}+R_{k} e^{i \theta}, k=\overline{1, n}$; 
b) domains $\Omega_{n k}, k=\overline{1, n}$, belong to Smirnov class, where $\Omega_{n k}$ is a simply connected domain with the boundary $\Gamma_{k}=f\left(\gamma_{k}\right)$ and $\Omega_{n k} \supset \Omega_{n}, \bigcap_{k=1}^{n} \Omega_{n k}=\Omega_{n}$.

Representation (1.1) just expresses that $f^{\prime}(z) \neq 0$ and $f^{\prime} \in H_{1}\left(D_{1}\right)$, and this is why the function $f$ can be non univalent in $D_{1} \equiv D$ also in the case $\mu(\theta) \equiv 0$. There are numerous studies providing various additional restrictions for $\ln \left|f^{\prime}\left(e^{i \theta}\right)\right|$ as $\mu(\theta) \equiv 0$ to ensure the univalence for $f$ in $D_{1}$. For the case $\mu(\theta) \not \equiv 0$, in papers [4], [7] and [8] there was established the relation between global univalence $f$ with the belonging of $\nu$ to the Zygmund class $\Lambda(K)$, that is, $|\nu(\theta+h)-2 \nu(\theta)+\nu(\theta-h)| \leqslant K h$; the relation was expressed in terms of restrictions for the constant $K>0$.

Following G.Ts. Tumarkin [16] and D. Khavinson [17], we obtain a convenient for us formula recovering the values of the function $g=f^{\prime}$ in the class $H_{1}\left(D_{n}\right)$ by a known up to a zero set measure absolute value of the boundary values $\Phi_{k}(\theta)=\left|g\left(\zeta_{k}(\theta)\right)\right|, \zeta_{k}(\theta)=a_{k}+R_{k} e^{i \theta}, k=\overline{1, n}$. We note that in the simply-connected case $n=1$ the proof of the structural formula for the class $H_{1}$ was given by V.I. Smirnov and is related with constructing the best possible analytic majorant for some family of analytic functions. This result on constructing the majorants was generalized by G.Ts. Tumarkin [16]. We describe briefly the main result. Let $H$ be a family of functions $g$ analytic in a finitely-connected domain $\Omega_{n}, \Omega_{n}^{j}$ be a sequence of domains converging to $\Omega$ as $j \rightarrow \infty$ such that

$$
\int_{\Gamma^{j}} \sup _{g \in H} \ln ^{+}|g(z)| \frac{\partial G_{j}\left(z, z_{0}\right)}{\partial n} d s \leqslant C<\infty, \quad j=1,2, \ldots
$$

Here $\Gamma^{j}$ is the boundary of the domain $\Omega_{n}^{j}, G_{j}\left(z, z_{0}\right)$ is the Green function of the domain $\Omega_{n}^{j}$ with the pole at a point $z_{0} \in \Omega_{n}^{j}, j=1,2, \ldots$ we denote by $u$ the best possible harmonic majorant of subharmonic function $\sup _{g \in H} \ln |g(z)|$. Let $v$ be the conjugate harmonic function. Then, if $\tilde{g}(z)=\exp \{u(z)+i v(z)\}$ is single-valued in $\Omega_{n}$, it is the best possible analytic majorant of the family $H$. If the constructed function $\tilde{g}$ is multiple-valued, the best possible analytic majorant does not exist. Let us consider the class $H_{1}\left(D_{n}\right)$ from this point of view. Condition (2.1) ensures inequality (2.2). Therefore, there exists the best possible harmonic majorant $u$, but at that, the constructed function $\tilde{g}$ can turn out to be multi-valued. We consider non-negative functions $\Phi_{1}, \Phi_{2}, \ldots, \Phi_{n}$ satisfying the conditions

$$
\begin{aligned}
& \Phi_{k} \in L^{1}[-\pi, \pi], \quad \ln \Phi_{k} \in L^{1}[-\pi, \pi], \\
& \sum_{j \neq k} \frac{1}{2 \pi} \int_{-\pi}^{\pi} \Phi_{j}(\theta) P_{j}\left(z_{k}, z_{j}(\theta)\right) d \theta=A, \quad k=\overline{1, n}, \quad A=\text { const },
\end{aligned}
$$

where $P_{j}\left(z, z_{j}\right)=\operatorname{Re} S_{j}\left(z, z_{j}\right),\left\{S_{j}\left(z, z_{j}\right)\right\}_{j=1}^{n}$ is the Schwarz kernel for the circular domain $D_{n}$ proposed by V.A. Zmorovich (see, for instance, [17]). By $\Phi\left(D_{n}\right)$ we denote the subclass $H_{1}\left(D_{n}\right)$ of functions $g$ with the single-valued logarithm such that almost everywhere in $\gamma$, the inequality $\left|g_{k}\left(z_{k}(\theta)\right)\right| \leqslant \Phi_{k}(\theta), k=\overline{1, n}$, holds true. The best possible harmonic majorant for $\sup _{g \in \Phi\left(D_{n}\right)}\{\ln |g(z)|\}$ is

$$
u(z)=\sum_{k=1}^{n} \frac{1}{2 \pi} \int_{-\pi}^{\pi} \Phi_{k}(\theta) P_{k}\left(z, z_{k}(\theta)\right) d \theta-A, z \in D_{n} .
$$


By relation (2.3), the analytic function $\tilde{g}$ defined by the identity

$$
\tilde{g}(z)=\exp \left\{\sum_{k=1}^{n} \frac{1}{2 \pi} \int_{-\pi}^{\pi} \Phi_{k}(\theta) S_{k}\left(z, z_{k}(\theta)\right) d \theta-A\right\} .
$$

is single-valued in $D_{n}$. Its logarithm $\ln \tilde{g}(\zeta)$ is also single-valued.

Assume now that $g \in H_{1}\left(D_{n}\right)$. It is known that almost everywhere in $\gamma_{k}$ there exist the angular boundary values $\Phi_{k}(\theta)=\left|g_{k}\left(z_{k}(\theta)\right)\right|$ and $\Phi_{k}$ and $\ln \Phi_{k}$ are summable functions. To transform the analytic function $\ln g$ to the single-valued form, we consider the function defined by the identity

$$
\Theta(z)=\prod_{k=1}^{n}\left[\Theta_{k}(z)\right]^{-\alpha_{k}}
$$

Here $\Theta_{k}$ maps conformally but not univalently the domain $D_{n}$ on the domain $q_{k}<|w|<1$ and the circle $|w|=q_{k}$ is in one-to-one correspondence with the circle $\gamma_{k}$. As it was proved in work [15], there exists the unique choice of numbers $\delta, \alpha_{k}$, such that $\ln F, F(z)=z^{-\delta} g(z) \Theta(z)$, is a single-valued analytic function. The function $F$ possesses angular boundary values $\left|F\left(z_{k}(\theta)\right)\right|=$ $\left|z_{k}(\theta)\right|^{-\delta} \Phi_{k}(\theta) q_{k}^{-\alpha_{k}}$ almost everywhere in $\gamma_{k}$ and these values satisfy conditions $(2.3)$. Therefore, $F$ can be represented as $F(z)=e^{i \beta} \tilde{g}(z) \sigma(z)$, where $\tilde{g}$ is the function of the maximal absolute value (2.4), a holomorphic in $D_{n}$ function $\ln \sigma$ belongs to the Carathéodory class and hence, it can be represented by the formula

$$
\sigma(z)=\exp \left\{\sum_{k=1}^{n} \frac{1}{2 \pi} \int_{-\pi}^{\pi} S_{k}\left(z, z_{k}(\theta)\right) d \nu_{k}(\theta)-B\right\}, \quad B=\sum_{j \neq k} \frac{1}{2 \pi} \int_{-\pi}^{\pi} P_{j}\left(z_{k}, z_{j}(\theta)\right) d \nu_{j}(\theta),
$$

$k=\overline{1, n}, \nu_{j}(\theta)$ is a non-decreasing singular function. Thus, the following statement holds true: each function $g=f^{\prime}$ in the class $H_{1}\left(D_{n}\right)$ can be represented as

$$
g(z)=e^{i \beta} z^{\delta} \Theta^{-1}(z) \tilde{g}(z) \sigma(z),
$$

where $\sigma$ is a holomorphic in $D_{n}$ function of form (2.5), $\tilde{g}$ is a function with representation (2.4) maximal for the set $\left|z_{k}(\theta)\right|^{-\delta} \Phi_{k}(\theta) q_{k}^{-\alpha_{k}}, k=\overline{1, n}, z^{\delta} \Theta^{-1}(z)$ selects the multi-valued part of $\ln g(z)$.

\section{CONDITIONS OF UNIVALENCE FOR THE MAPPING OF AN ANNULUS}

Assume that the function $f$ is holomorphic in the annulus $D_{2} \equiv E(q, 1)=\{z \in \mathrm{C}: q<$ $|z|<1\}$ and can be continuously continued to the circumference $|\zeta|=q \in(0,1)$. Suppose that $f^{\prime} \in H_{1}(E(q, 1)), f^{\prime}(z) \neq 0$ for each point $z \in E(q, 1)$. Then for each point $z \in E(q, 1)$

$$
f(z)=\int \exp \left\{\frac{1}{2 \pi} \int_{-\pi}^{\pi} P(\theta) S_{q}\left(q z^{-1} e^{i \theta}\right) d \theta+\frac{1}{2 \pi} \int_{-\pi}^{\pi} S_{q}\left(z e^{-i \theta}\right) d \mu(\theta)-A\right\} d z .
$$

Here $S_{q}$ is the Ville kernel, that is,

$$
S_{q}(z)=\frac{1+z}{1-z}+\sum_{\nu=1}^{\infty} \frac{1+q^{2 \nu} z}{1-q^{2 \nu} z}-\frac{1+q^{2 \nu} z^{-1}}{1-q^{2 \nu} z^{-1}}
$$

We suppose that the relation

$$
\frac{1}{2 \pi} \int_{-\pi}^{\pi} P(\theta) d \theta=\frac{1}{2 \pi} \int_{-\pi}^{\pi} d \mu(\theta)=A=\text { const }
$$


holds true, where $\mu$ is a non-increasing singular function with a derivative vanishing almost everywhere, and $P$ is a continuous $2 \pi$-periodic function. The singular function $\mu$ is continued to the entire real line by the identity:

$$
\mu(\theta+2 \pi)=\mu(\theta)-2 \pi \beta, \quad \theta \in \mathbb{R}, \quad \text { where } \quad \beta=-(2 \pi)^{-1} \int_{0}^{2 \pi} d \mu(\theta) \geqslant 0 .
$$

Let $\alpha \in(0,1), K$ be a positive constant. We consider the following three classes of real-valued functions:

a) $\lambda(K)$ is the class of all $2 \pi$-periodic functions $P$ satisfying the Lipschitz condition $\mid P(\theta+$ h) $-P(\theta) \mid \leqslant K$, where $\theta, h \in \mathbb{R}$;

b) $\Lambda_{1+\alpha}(K)$ is the class of all $2 \pi$-periodic functions $P$ satisfying the Zygmund condition of order $1+\alpha \in(1,2)$ :

$$
|P(\theta+h)-2 P(\theta)+P(\theta-h)| \leqslant K h^{1+\alpha} \quad \forall \theta, h \in \mathbb{R} ;
$$

c) $\Lambda(K)$ is the class of all singular functions $\mu$ satisfying the Zygmund condition of order 1:

$$
|\mu(\theta+h)-2 \mu(\theta)+\mu(\theta-h)| \leqslant K h \quad \theta, h \in \mathbb{R} .
$$

Let us obtain conditions for positive numbers $q, \beta$ and $K$ ensuring the univalence for mapping (3.1). We shall need the inequalities

$$
\begin{aligned}
& \frac{4 q \beta}{(1-q)^{2}}+\frac{K}{1-q^{2}}\left[\left(\frac{\pi}{2}\right)^{\alpha} 2 q+(1+2 q) B(\alpha)\right]<1 \\
& \frac{4 q \beta}{(1-q)^{2}}+K\left[1+\frac{\left(3-q^{2}\right)}{(1-q)^{2}}\left(\left(\frac{\pi}{2}\right)^{\alpha} q+B(\alpha)\right)+\left(6,6+\frac{17,3 q}{1-q^{2}}\right) \frac{1+1,8 q}{1-q^{2}}\right]<1
\end{aligned}
$$

where

$$
B(\alpha)=\frac{1+\alpha}{\pi} \int_{0}^{\pi} \frac{t^{\alpha}}{\sin t} d t
$$

Theorem 3.1. Let $\alpha, q$ be numbers in the interval $(0,1)$. Let $f$ be the holomorphic function defined by integral representation (3.1) in the annulus $E(q, 1)$, in which $P \in \Lambda_{1+\alpha}(K)$ and $\mu \in \Lambda(K)$ for some $K>0$. The function $f$ is an univalent conformal mapping of $E(q, 1)$ if the numbers $K>0$ and $q \beta>0$ are small enough, namely, if they satisfy inequalities (3.2) and (3.3).

In order to prove the main result of this section, we shall need a series of auxiliary estimates. Let

$$
g_{1}(z)=\frac{1}{2 \pi} \int_{-\pi}^{\pi} P(\theta) S_{q}\left(q z^{-1} e^{i \theta}\right) d \theta, \quad g_{2}(z)=\frac{1}{2 \pi} \int_{-\pi}^{\pi} S_{q}\left(z e^{-i \theta}\right) d \mu(\theta), \quad z \in E(q, 1) .
$$

Lemma 3.1. Let $z=r e^{i \varphi} \in E(q, 1)$. If $P \in \lambda(K)$, then the inequality

$$
\left|\operatorname{Re} \frac{\partial}{\partial \varphi} g_{1}\left(r e^{i \varphi}\right)\right| \leqslant K
$$

holds true. If $P \in \Lambda_{1+\alpha}(K)$, then

$$
\left|\operatorname{Im} \frac{\partial}{\partial \varphi} g_{1}\left(r e^{i \varphi}\right)\right| \leqslant \frac{K\left(3-q^{2}\right)}{1-q^{2}}\left[\left(\frac{\pi}{2}\right)^{\alpha} q+B(\alpha)\right], \quad B(\alpha)=\frac{1+\alpha}{\pi} \int_{0}^{\pi} \frac{t^{\alpha}}{\sin t} d t .
$$


Proof. Letting

$$
\begin{array}{ll}
S_{q}\left(r e^{i t}\right)=P_{q}(r, t)+i Q_{q}(r, t), & P_{q}(r, t)=P(r, t)+P_{q}^{0}(r, t), \\
P(r, t)=\frac{1-r^{2}}{1+r^{2}-2 r \cos t}, & P_{q}^{0}(r, t)=\sum_{j=1}^{\infty}\left[P\left(q^{2 j} r, t\right)-P\left(q^{2 j} / r, t\right)\right],
\end{array}
$$

for a harmonic in $E(q, 1)$ function $\frac{\partial}{\partial \varphi} \operatorname{Re} g_{1}(z), z=r e^{i \varphi}$, we obtain the representation

$$
\frac{\partial}{\partial \varphi} \operatorname{Re} g_{1}(z)=-\frac{1}{2 \pi} \int_{-\pi}^{\pi} P(\varphi+t) \frac{\partial}{\partial t} P_{q}(q / r, t) d t
$$

in which we denote $t=\theta-\varphi$. Replacing $t$ by $-t$, in view of the oddness of the function $\frac{\partial}{\partial t} P_{q}(q / r, t)$ we obtain

$$
\frac{\partial}{\partial \varphi} \operatorname{Re} g_{1}(z)=\frac{1}{2 \pi} \int_{-\pi}^{\pi} P(\varphi-t) \frac{\partial}{\partial t} P_{q}(q / r, t) d t
$$

Therefore,

$$
\frac{\partial}{\partial \varphi} \operatorname{Re} g_{1}(z)=-\frac{1}{2 \pi} \int_{-\pi}^{\pi}[P(\varphi+t)-P(\varphi-t)] \frac{\partial}{\partial t} P_{q}(q / r, t) d t .
$$

Employing the inequality $\frac{\partial}{\partial t} P_{q}(r, t)<0[24]$, we estimate the absolute value:

$$
\left|\frac{\partial}{\partial \varphi} \operatorname{Re} g_{1}(z)\right| \leqslant \frac{K}{\pi} \int_{0}^{\pi} t\left|\frac{\partial}{\partial t} P_{q}(q / r, t)\right| d t=-K P_{q}(q / r, \pi)+\frac{K}{\pi} \int_{0}^{\pi} P_{q}(q / r, t) d t .
$$

To complete the proof of the first statement of the lemma it remains to observe that

$$
\int_{0}^{\pi} P_{q}(\rho, t) d t=\pi, q<\rho<1 .
$$

Assume that $P \in \Lambda_{1+\alpha}(K)$. It is easy to check the identity

$$
\frac{\partial}{\partial \varphi} \operatorname{Im} g_{1}(z)=-\frac{1}{2 \pi} \int_{-\pi}^{\pi} P(\theta) \frac{\partial}{\partial \theta} Q_{q}(q / r, \theta-\varphi) d \theta
$$

where

$$
\begin{aligned}
& Q_{q}(\rho, t)=Q(\rho, t)+Q_{q}^{0}(\rho, t), \quad Q(\rho, t)=\frac{2 \rho \sin t}{1+\rho^{2}-2 \rho \cos t} \\
& Q_{q}^{0}(\rho, t)=\sum_{\nu=1}^{\infty} Q\left(q^{2 \nu} \rho, t\right)+Q\left(q^{2 \nu} / \rho, t\right)
\end{aligned}
$$

Employing the oddness of the function $\frac{\partial}{\partial t} Q_{q}(q / r, t)$ and the identity

$$
\int_{-\pi}^{\pi} \frac{\partial}{\partial t} Q_{q}(\rho, t) d t=0, q<\rho<1
$$


we transform the formula for $\frac{\partial}{\partial \varphi} \operatorname{Im} g_{1}(z)$ to the form

$$
\frac{\partial}{\partial \varphi} \operatorname{Im} g_{1}(z)=-\frac{1}{4 \pi} \int_{-\pi}^{\pi}[P(\varphi+t)-2 P(\varphi)+P(\varphi-t)] \frac{\partial}{\partial t} Q_{q}(q / r, t) d t .
$$

This implies the inequality

$$
\left|\frac{\partial}{\partial \varphi} \operatorname{Im} g_{1}(z)\right| \leqslant \frac{K}{2 \pi} \int_{0}^{\pi} t^{1+\alpha}\left|\frac{\partial}{\partial t} Q_{q}(q / r, t)\right| d t
$$

which we represent as

$$
\begin{aligned}
& \left|\frac{\partial}{\partial \varphi} \operatorname{Im} g_{1}(z)\right| \leqslant \frac{K}{2 \pi}\left[I_{1}(q / r, \alpha)+I_{2}(q / r, \alpha)\right] \\
& I_{1}(\rho, \alpha)=\int_{0}^{\pi} t^{1+\alpha}\left|\frac{\partial}{\partial t} Q(\rho, t)\right| d t, \quad I_{2}(\rho, \alpha)=\int_{0}^{\pi} t^{1+\alpha}\left|\frac{\partial}{\partial t} Q_{q}^{0}(\rho, t)\right| d t \\
& \frac{\partial}{\partial t} Q(\rho, t)=\frac{\left(1+\rho^{2}\right) 2 \rho \cos t-4 \rho^{2}}{\left(1+\rho^{2}-2 \rho \cos t\right)^{2}}, \quad q<\rho<1, \\
& \frac{\partial}{\partial t} Q_{q}^{0}(q / r, t)=\sum_{\nu=1}^{\infty}\left[\frac{\partial}{\partial t} Q\left(q^{2 \nu} r, t\right)+\frac{\partial}{\partial t} Q\left(q^{2 \nu} / r, t\right)\right] .
\end{aligned}
$$

We rewrite the formula for $I_{1}(\rho, \alpha)$ as

$$
I_{1}(\rho, \alpha)=\int_{0}^{\tau} t^{1+\alpha} \frac{\partial}{\partial t} Q(\rho, t) d t-\int_{\tau}^{\pi} t^{1+\alpha} \frac{\partial}{\partial t} Q(\rho, t) d t
$$

where $\tau=\arcsin \left(1-\rho^{2}\right) /\left(1+\rho^{2}\right)$. By the integration by parts we obtain

$$
I_{1}(\rho, \alpha)=2 \tau^{1+\alpha} Q(\rho, \tau)+(1+\alpha) \int_{\tau}^{\pi} t^{\alpha} Q(\rho, t) d t-(1+\alpha) \int_{0}^{\tau} t^{\alpha} Q(\rho, t) d t .
$$

By the identity $Q(\rho, \tau)=2 \rho /\left(1-\rho^{2}\right)$ we get

$$
I_{1}(\rho, \alpha) \leqslant 2 \rho \frac{\pi^{1+\alpha}\left(1-\rho^{2}\right)^{\alpha}}{2^{\alpha}\left(1+\rho^{2}\right)^{1+\alpha}}+(1+\alpha) \int_{0}^{\pi} t^{\alpha} Q(\rho, t) d t .
$$

Letting $\rho=q / r$ in $(3.6)$ and estimating the right hand side by means with the the inequality $Q(\rho, t)<2 \rho / \sin t$, we obtain

$$
I_{1}(q / r, \alpha)<2 \pi\left[\left(\frac{\pi}{2}\right)^{\alpha} q+B(\alpha)\right] .
$$

In order to estimate $I_{2}(q / r, \alpha)$, we observe that series 3.5 converges absolutely and uniformly and hence, we can write the inequality

$$
I_{2}(q / r, \alpha)<\sum_{\nu=1}^{\infty} \int_{0}^{\pi} t^{1+\alpha}\left|\frac{\partial}{\partial t} Q\left(q^{2 \nu} r, t\right)\right| d t+\sum_{\nu=1}^{\infty} \int_{0}^{\pi} t^{1+\alpha}\left|\frac{\partial}{\partial t} Q\left(q^{2 \nu} / r, t\right)\right| d t .
$$

By this inequality and (3.6) we obtain the estimate

$$
I_{2}(q / r, \alpha)<\frac{\pi^{1+\alpha}}{2^{\alpha}} \sum_{\nu=1}^{\infty} q^{2 \nu} \frac{\left(1-q^{4 \nu+2}\right)^{\alpha}}{\left(1+q^{4 \nu+2}\right)^{1+\alpha}}+\frac{\pi^{1+\alpha}}{2^{\alpha}} \sum_{\nu=1}^{\infty} q^{2 \nu-1} \frac{\left(1-q^{4 \nu}\right)^{\alpha}}{\left(1+q^{4 \nu}\right)^{1+\alpha}}
$$




$$
+(1+\alpha) \sum_{\nu=1}^{\infty} \int_{0}^{\pi} t^{\alpha} Q\left(q^{2 \nu} r, t\right) d t+(1+\alpha) \sum_{\nu=1}^{\infty} \int_{0}^{\pi} t^{\alpha} Q\left(q^{2 \nu} / r, t\right) d t .
$$

This implies the relation

$$
I_{2}(q / r, \alpha)<\frac{4 \pi q}{1-q^{2}}\left[\left(\frac{\pi}{2}\right)^{\alpha}+B(\alpha)\right]
$$

Substituting the latter inequality and inequality (3.7) into (3.4), we arrive at the second statement of the lemma.

Lemma 3.2. Let $z=r e^{i \varphi} \in E(q, 1)$. If $\mu \in \Lambda(K)$, then the identity

$$
\left|\operatorname{Re} \frac{\partial}{\partial \varphi} g_{2}\left(r e^{i \varphi}\right)\right| \leqslant K \frac{0,4(1+r)+2,1 q /(1-q)}{1-r}
$$

holds true.

Proof. We take the derivative w.r.t. $\varphi$ of the both sides of the identity

$$
\operatorname{Re} g_{2}(z)=-\frac{1}{2 \pi} \int_{-\pi}^{\pi} \mu(\theta) \frac{\partial}{\partial \theta} P_{q}(r, \theta-\varphi) d \theta, \quad z=r e^{i \varphi}
$$

and obtain

$$
\frac{\partial}{\partial \varphi} \operatorname{Re} g_{2}(z)=\frac{1}{2 \pi} \int_{-\pi}^{\pi} \mu(\theta) \frac{\partial^{2}}{\partial \theta^{2}} P_{q}(r, \theta-\varphi) d \theta, \quad z=r e^{i \varphi}
$$

Employing the evenness of the function $\frac{\partial^{2}}{\partial \theta^{2}} P_{q}(r, \theta-\varphi)$, we represent the latter formula as

$$
\frac{\partial}{\partial \varphi} \operatorname{Re} g_{2}(z)=\frac{1}{2 \pi} \int_{0}^{\pi}[\mu(\varphi+t)-2 \mu(\varphi)+\mu(\varphi-t)] \frac{\partial^{2}}{\partial t^{2}} P_{q}(r, t) d t, \quad z=r e^{i \varphi} .
$$

Since the function $\mu$ belongs to the class $\Lambda(K)$, we obtain the inequality

$$
\left|\frac{\partial}{\partial \varphi} \operatorname{Re} g_{2}(z)\right| \leqslant \frac{K}{2 \pi} \int_{0}^{\pi} t\left|\frac{\partial^{2}}{\partial t^{2}} P_{q}(r, t)\right| d t
$$

Using the expansion

we write

$$
\frac{\partial^{2}}{\partial t^{2}} P_{q}(r, t)=\frac{\partial^{2}}{\partial t^{2}} P(r, t)+\frac{\partial^{2}}{\partial t^{2}} P_{q}^{0}(r, t)
$$

where

$$
\left|\frac{\partial}{\partial \varphi} \operatorname{Re} g_{2}(z)\right| \leqslant I_{1}(r)+I_{2}(r), \quad z=r e^{i \varphi},
$$

$$
I_{1}(r)=\frac{K}{2 \pi} \int_{0}^{\pi} t\left|\frac{\partial^{2}}{\partial t^{2}} P(r, t)\right| d t, \quad I_{2}(r)=\frac{K}{2 \pi} \int_{0}^{\pi} t\left|\frac{\partial^{2}}{\partial t^{2}} P_{q}^{0}(r, t)\right| d t .
$$

Following work [7], we obtain the inequalities

$$
I_{1}(r) \leqslant \frac{0,4 K(1+r)}{1-r}, \quad I_{1}(r) \leqslant r \frac{2,1 K}{1-r} .
$$

Taking into consideration the formula

$$
\frac{\partial^{2}}{\partial t^{2}} P_{q}^{0}(r, t)=\sum_{j=1}^{\infty}\left[\frac{\partial^{2}}{\partial t^{2}} P\left(r q^{2 j}, t\right)-\frac{\partial^{2}}{\partial t^{2}} P\left(q^{2 j} / r, t\right)\right]
$$


in which the series are majorized by the geometric progression with the general term $12 q^{2 j-1} /(1-q)^{3}$ and they hence converge absolutely and uniformly for $q \leqslant r \leqslant 1$, we obtain the following inequality for $I_{2}(r)$ :

$$
I_{2}(r) \leqslant \sum_{j=1}^{\infty}\left(\frac{K}{2 \pi} \int_{0}^{\pi} t\left|\frac{\partial^{2}}{\partial t^{2}} P\left(r q^{2 j}, t\right)\right| d t+\frac{K}{2 \pi} \int_{0}^{\pi} t\left|\frac{\partial^{2}}{\partial t^{2}} P\left(q^{2 j} / r, t\right)\right| d t\right) .
$$

Employing the second inequality in $(3.8)$, we write

$$
I_{2}(r) \leqslant 2,1 K \sum_{j=1}^{\infty}\left(\frac{r q^{2 j}}{1-r q^{2 j}}+\frac{q^{2 j} / r}{1-q^{2 j} / r}\right) \leqslant 2,1 K \sum_{j=1}^{\infty} \frac{q^{j}}{1-q^{j}}<\frac{2,1 K q}{(1-q)^{2}} .
$$

By inequality (3.8) this implies the statement of the lemma.

Lemma 3.3. Let a function $F$ be holomorphic in the annulus $E(q, 1)$, be continuously continuable on the boundary circumference $|z|=q$ and have the zero real part on the circumference $|z|=q$. If the inequality $\left|\operatorname{Re} F\left(r e^{i \varphi}\right)\right| \leqslant \frac{C}{(1-r)}$ holds in the annulus $E(q, 1)$ with some constant $C$, then

$$
|F(z)| \leqslant \frac{8,2 C(1+1,8 q)}{(1-r)\left(1-q^{2}\right)}+\max _{\varphi \in[-\pi, \pi]}\left|\operatorname{Im} F\left(q e^{i \varphi}\right)\right| .
$$

Proof. Let $\rho$ be some number obeying the condition $q<\rho<1, \tilde{q}=q / \rho$. The function $F$ is holomorphic in the annulus $E(\tilde{q}, \rho) \subset E(q, 1)$ and is continuously continuable on the boundary circumference $|\zeta|=\rho$. First we estimate the absolute value of the function $F$, which we write as

$$
F^{\prime}(z)=\frac{1}{2 \pi} \int_{-\pi}^{\pi} \operatorname{Re} F\left(\rho e^{i \theta}\right)\left[\frac{d}{d z} S\left(\rho^{-1} z e^{-i \theta}\right)+\frac{d}{d z} S_{\tilde{q}}^{0}\left(\rho^{-1} z e^{-i \theta}\right)\right] d \theta .
$$

Since

$$
\begin{gathered}
\left|\frac{d}{d z} S\left(\rho^{-1} z e^{-i \theta}\right)\right|=\frac{2 \rho}{\rho^{2}-r^{2}} P(r / \rho, \theta-\varphi), \\
\left|\frac{d}{d z} S_{\tilde{q}}^{0}\left(\rho^{-1} z e^{-i \theta}\right)\right| \leqslant \sum_{j=1}^{\infty}\left[\frac{2 \rho \tilde{q}^{2 j}}{\rho^{2}-r^{2} \tilde{q}^{4 j}} P\left(r \tilde{q}^{2 j} / \rho, \theta-\varphi\right)+\frac{2 \rho \tilde{q}^{2 j}}{r^{2}-\rho^{2} \tilde{q}^{4 j}} P\left(\rho \tilde{q}^{2 j} / r, \theta-\varphi\right)\right],
\end{gathered}
$$

estimating the absolute values in the representation for $F^{\prime}(z)$, we obtain

$$
\left|F^{\prime}(z)\right| \leqslant \frac{C}{1-\rho}\left[\frac{2 \rho}{\rho^{2}-r^{2}}+\sum_{j=1}^{\infty} \frac{2 \rho \tilde{q}^{2 j}}{\rho^{2}-r^{2} \tilde{q}^{4 j}}+\frac{2 \rho \tilde{q}^{2 j}}{r^{2}-\rho^{2} \tilde{q}^{4 j}}\right] .
$$

Taking into consideration the inequalities

$$
\begin{aligned}
& \sum_{j=1}^{\infty} \frac{2 \rho \tilde{q}^{2 j}}{\rho^{2}-r^{2} \tilde{q}^{4 j}} \leqslant \frac{2 \rho}{\rho^{2}-r^{2}} \sum_{j=1}^{\infty} \tilde{q}^{2 j}=\frac{2 \rho q^{2}}{\left(\rho^{2}-r^{2}\right)\left(\rho^{2}-q^{2}\right)}, \\
& \sum_{j=1}^{\infty} \frac{2 \rho \tilde{q}^{2 j}}{r^{2}-\rho^{2} \tilde{q}^{4 j}} \leqslant \frac{2 \rho}{r^{2}\left(1-\tilde{q}^{2}\right)} \sum_{j=1}^{\infty} \tilde{q}^{2 j} \leqslant \frac{2 \rho q^{2}}{r^{2}\left(\rho^{2}-r^{2}\right)\left(\rho^{2}-q^{2}\right)},
\end{aligned}
$$

we obtain the following estimate for $\left|F^{\prime}(z)\right|$ :

$$
\left|F^{\prime}(z)\right| \leqslant \frac{2 C \rho}{(1-\rho)\left(\rho^{2}-r^{2}\right)}\left[1+\frac{q^{2}}{\rho^{2}-q^{2}}+\frac{q^{2}}{r^{2}\left(\rho^{2}-q^{2}\right)}\right] .
$$


Choosing $\rho=(1+\sqrt{2} r) /(1+\sqrt{2})$ in this inequality, we obtain

$$
\left|F^{\prime}(z)\right| \leqslant \frac{8,2 C}{(1-r)^{2}}\left[1+\frac{q^{2}}{1-q^{2}}+\frac{q^{2}}{r^{2}\left(1-q^{2}\right)}\right] .
$$

Integrating the latter inequality w.r.t. the radius, we obtain the statement of the lemma.

Proof of Theorem 3.1. We consider the mapping of the annulus $E(q, 1)$ by function (3.1). We assume that $P \in \Lambda_{1+\alpha}(K)$. The necessary and sufficient condition for the convexity of the curve $\Gamma_{q}$, which the image of the circumference $\gamma_{q}=\left\{\zeta, \zeta=q e^{i \theta},-\pi \leqslant \theta \leqslant \pi\right\}$ under the mapping by the function $f$, is the inequality

$$
\lim _{z \rightarrow \zeta} \operatorname{Re} z \frac{f^{\prime \prime}(z)}{f^{\prime}(z)}>-1, \zeta \in \gamma_{q}
$$

By formula (3.1) we obtain

$$
i z \frac{f^{\prime \prime}(z)}{f^{\prime}(z)}=\frac{\partial}{\partial \varphi} g_{1}(z)+\frac{\partial}{\partial \varphi} g_{2}(z), z=r e^{i \varphi} .
$$

This implies the representation

$$
i \zeta \frac{f^{\prime \prime}(\zeta)}{f^{\prime}(\zeta)}=\frac{\partial}{\partial \varphi} g_{1}(\zeta)+\frac{\partial}{\partial \varphi} g_{2}(\zeta), \zeta=q e^{i \varphi} .
$$

To ensure condition (3.9), it is sufficient to satisfy the inequalities

$$
\left|\operatorname{Im} \frac{\partial}{\partial \varphi} g_{1}(\zeta)+\operatorname{Im} \frac{\partial}{\partial \varphi} g_{2}(\zeta)\right|<1
$$

Due to the inequality

for the function

$$
\frac{\partial}{\partial t} Q_{q}\left(q e^{i t}\right)<4 q /(1-q)^{2}
$$

$$
\left|\frac{\partial}{\partial \varphi} g_{2}(\zeta)\right|=\frac{1}{2 \pi} \int_{-\pi}^{\pi} \frac{\partial}{\partial \varphi} Q_{q}\left(q e^{i(\varphi-\theta)}\right) d \mu(\theta), \quad \zeta=q e^{i \varphi}
$$

we obtain

$$
\left|\frac{\partial}{\partial \varphi} g_{2}(\zeta)\right| \leqslant \frac{4 q}{(1-q)^{2}} \beta, \quad \zeta=q e^{i \varphi}, \quad \beta=\frac{1}{2 \pi} \int_{-\pi}^{\pi}|d \mu(\theta)| .
$$

In view of inequality 3.6 , by Lemma 1 , for the function $\frac{\partial}{\partial \varphi} g_{1}(\zeta)$ we have

$$
\left|\operatorname{Im} \frac{\partial}{\partial \varphi} g_{1}(\zeta)\right| \leqslant \frac{K}{1-q^{2}}\left[\left(\frac{\pi}{2}\right)^{\alpha} 2 q+(1+2 q) B(\alpha)\right] .
$$

Therefore, inequality 3.10 holds by condition 3.2 .

By formula (3.1) we obtain the inequality

$$
\left|z \frac{f^{\prime \prime}(z)}{f^{\prime}(z)}\right|<\left|\frac{\partial}{\partial \varphi} g_{1}(z)\right|+\left|\frac{\partial}{\partial \varphi} g_{2}(z)\right|, \quad z=r e^{i \varphi} \in E(q, 1) .
$$

Employing the above obtained estimates, we write the inequality

$$
\left|z \frac{f^{\prime \prime}(z)}{f^{\prime}(z)}\right|<\frac{4 q \beta}{(1-q)^{2}}+K\left[1+\frac{\left(3-q^{2}\right)}{(1-q)^{2}}\left(\left(\frac{\pi}{2}\right)^{\alpha} q+B(\alpha)\right)+\left(6,6+\frac{17,3 q}{1-q^{2}}\right) \frac{1+1,8 q}{1-q^{2}}\right],
$$

which together with condition $(3.3)$ yields the inequality $\left|z f^{\prime \prime}(z) / f^{\prime}(z)\right|<\left(1-|z|^{2}\right)^{-1}$ for each point $z \in E(q, 1)$. 
To complete the proof of Theorem 3.1 for a holomorphic in the annulus $E(q, 1)$ function $f$, we establish a sufficient condition for the univalence by the continuation method presented in [18], see the proposition after this proof. This condition completes the prove of the theorem.

Proposition. Let a function $f$ be holomorphic in $E(q, 1)$, the harmonic function $\operatorname{Re}\left[z f^{\prime \prime}(z) / f^{\prime}(z)\right]$ be continuously continuable on the internal boundary $|z|=q$. If

$$
\begin{aligned}
& \left|z f^{\prime \prime}(z) / f^{\prime}(z)\right|<1 /\left(1-|z|^{2}\right), \quad z \in E(q, 1), \\
& \int_{-\pi}^{\pi} \operatorname{Re}\left[1+\zeta f^{\prime \prime}(\zeta) / f^{\prime}(\zeta)\right] d \theta=2 \pi, \quad \operatorname{Re}\left[1+\zeta f^{\prime \prime}(\zeta) / f^{\prime}(\zeta)\right] \geqslant 0, \quad \zeta=q e^{i \theta},
\end{aligned}
$$

then the function $f$ is univalent in $E(q, 1)$.

Proof. By condition 3.12, the image of the circumference $\gamma_{q}:=\{|\zeta|=q\}$ under the mapping by the function $f$ is a closed convex curve $\Gamma_{q}$ passed once counterclockwise, when $\theta$ ranges from $-\pi$ to $\pi$. We choose a homeomorphism $g$ of the circle $\bar{E}_{q}:=\{z:|z| \leqslant q\}$ on the closed bounded domain with the boundary $\Gamma_{q}$ satisfying the gluing condition $f\left(q e^{i \theta}\right)=g\left(q e^{i \theta}\right)$. Let $d$ be some number $q<d<1$. We define the mapping of the entire plane as follows:

$$
\hat{f}(\zeta)= \begin{cases}g(\zeta), & \zeta \in \bar{E}_{q}, \\ f(\zeta), & \zeta \in E(q, d), \\ f\left(d^{2} / \bar{\zeta}\right)+\left(\zeta-\frac{d^{2}}{\bar{\zeta}}\right) f^{\prime}\left(\frac{d^{2}}{\bar{\zeta}}\right), & \zeta \in E\left(d, \frac{d^{2}}{q}\right), \\ f\left(q e^{i \theta}\right)+\left(1-\frac{q}{d^{2}}\right) r e^{i \theta} f^{\prime}\left(q e^{i \theta}\right), & \zeta \in \bar{E}_{d^{2} / q}^{-}:=\left\{\zeta:|\zeta| \geqslant d^{2} / q\right\} .\end{cases}
$$

The function $\hat{f}$ is locally univalent in the entire complex plane. Indeed, in $\bar{E}_{q}$, the function $\hat{f}(\zeta) \equiv g(\zeta)$ is univalent by the construction. As $\zeta \in \bar{E}(q, d)$, the inequality $\left|f^{\prime}(\zeta)\right|>0$ is implied by 3.11 . In the annulus $E\left(d, \frac{d^{2}}{q}\right)$, the condition for the local univalence for the function $\hat{f}$ is the inequality $I=\left|\hat{f}_{\zeta}\right|^{2}-\left|\hat{f}_{\bar{\zeta}}\right|^{2}>0$. We write an explicit expression for the Jacobian of the mapping $\hat{f}$ in the annulus $E\left(d, \frac{d^{2}}{q}\right)$

$$
I=\left|f^{\prime}\left(d^{2} / \bar{\zeta}\right)\right|^{2}\left[1-\left|\left(\zeta-\frac{d^{2}}{\bar{\zeta}}\right) \frac{d^{2}}{\bar{\zeta}^{2}}\right|^{2}\left|\frac{f^{\prime \prime}\left(d^{2} / \bar{\zeta}\right)}{f^{\prime}\left(d^{2} / \bar{\zeta}\right)}\right|^{2}\right] .
$$

It clearly yields that the positive orientation of the mapping is implied by condition 3.11. If $\zeta \in \bar{E}_{d^{2} / q}^{-}$, then the condition of the local univalence at this point is equivalent to the inequality

$$
1+\operatorname{Re} q e^{i \theta} \frac{f^{\prime \prime}\left(q e^{i \theta}\right)}{f^{\prime}\left(q e^{i \theta}\right)}+\frac{d^{2}}{r\left(d^{2}-q^{2}\right)}>0, \quad f^{\prime}\left(q e^{i \theta}\right) \neq 0, \quad r=|\zeta| \geqslant \frac{d^{2}}{q}
$$

implied by condition 3.12 .

Since the mapping $\hat{f}$ transforms each sequence of the points converging to infinity into a similar one, by the Hadamard theorem (see, for instance, [18], 21]), the local univalence of $\hat{f}$ implies the univalence in the entire plane. Thus, $f$ is univalent in the annulus $E(q, 1)$.

\section{Conditions of the univalence for mappings of Finitely-CONNECTED domains}

Let $f$ be a univalent conformal mapping of $D_{n}$ onto some domain $\Omega_{n} ; \Omega_{n k}$ be the simplyconnected domain introduced in Definition $2 \mathrm{~b}$ ). In the same way we introduce the domains $D_{n k}$. This is clearly a circle or the exterior of the circle with the boundary $\gamma_{k}$. We take a concentric 
with $\gamma_{k}$ circumference $\gamma_{k}^{\prime}, \gamma_{k}^{\prime}=\left\{\zeta:\left|\zeta-a_{k}\right|=R_{k}^{\prime}\right\}$ and by $D_{n k}^{\prime}$ we denote the circular annulus with the boundary $\gamma_{k} \cup \gamma_{k}^{\prime}$.

Lemma 4.1. The representation

$$
f(\zeta)=\left(f_{k} \circ w_{k}\right)(\zeta), \quad \zeta \in D_{n k}^{\prime},
$$

holds true, where $f_{k}$ is any of univalent mappings of $D_{n k}$ onto $\Omega_{n k}$, and $w_{k}$ is a homeomorphism of $D_{n k}$ onto itself conformal in $D_{n k}^{\prime}$.

Proof. We continue the mapping $f$ of the annulus $D_{n k}^{\prime}$ onto the two-connected domain $\Omega_{n k}^{\prime}$ to the homeomorphism $\hat{f}$ of the domain $D_{n k}$ onto $\Omega_{n k}$. For instance, this can be done as follows. We choose some function $\varphi_{k}$, which is a univalent conformal mapping of $D_{n k} \backslash D_{n k}^{\prime}$ onto the domain $\Omega_{n k} \backslash \Omega_{n k}^{\prime}$. Then as $\hat{f}$, we can take the function defined by the identities

$$
\hat{f}(\zeta)=\left\{\begin{array}{l}
f(\zeta), \quad \zeta \in E_{n k}^{\prime}, \\
\varphi\left(a_{k}+r e^{i \tau}\right), \quad \tau=\arg \left\{\varphi^{-1}\left[f\left(a_{k}+R_{k}^{\prime} e^{i \theta}\right)\right]-a_{k}\right\}, \quad \zeta=a_{k}+r e^{i \theta} \in E_{n k} \backslash E_{n k}^{\prime} .
\end{array}\right.
$$

The function $\hat{f}$ is a homeomorphic mapping of $D_{n k}$ onto $\Omega_{n k}$, and therefore, it is inner in Stoilov sense. By the Stoilov theorem, $\hat{f}(\zeta)=\left(F \circ w_{k}\right)(\zeta)$, where $w_{k}$ is the homeomorphism of $D_{n k}$ onto itself, the function $F$ is analytic in $D_{n k}$. Since $F\left(w_{k}\right)=(\hat{f} \circ \zeta)\left(w_{k}\right)$, then $F$ is univalent in $D_{n k}^{\prime}$, that is, $F(z)=f_{k}(z)$. The conformity of $w_{k}$ in $D_{n k}^{\prime}$ is implied immediately the identity $w_{k}(\zeta)=\left(f_{k}^{-1} \circ f\right)(\zeta), \zeta \in D_{n k}^{\prime}$. The proof is complete.

For further purposes, it is convenient to select a thin annulus $D_{n k}^{\prime \prime}$ located in the annulus $D_{n k}^{\prime}$. Namely, let $\gamma_{k}^{\prime \prime}=\left\{\zeta:\left|\zeta-a_{k}\right|=R_{k}^{\prime \prime}\right\}$ be located in $D_{n k}^{\prime}$. Then we let that $D_{n k}^{\prime \prime}$ is the annulus with the boundary $\gamma_{k} \bigcup \gamma_{k}^{\prime \prime}$. Taking into account the analyticity of $w_{k}$ on $\gamma_{k}$, by the Riemann-Schwarz symmetry principle we obtain that $w_{k}$ is a univalent analytic function in the closed domain $\bar{D}_{n k}^{\prime \prime}$. This is why all derivatives of $w_{k}(\zeta)$ and continuous and uniformly bounded in $\bar{D}_{n k}^{\prime \prime}$. And moreover, there exist positive constants $C_{j}$ such that in $\bar{D}_{n k}^{\prime \prime}$ the estimates

$$
C_{1} \leqslant\left|\ln w_{k}^{\prime}(\zeta)\right|<C_{2}, \quad C_{3} \leqslant\left(R_{k}-\left|\zeta-a_{k}\right|\right) /\left(R_{k}-\left|w_{k}-a_{k}\right|\right) \leqslant C_{4}
$$

hold true.

By means of the lemma we propose a simple proof of the equivalence of Definitions 2a) and $2 \mathrm{~b})$. Indeed, we have

$$
\ln f^{\prime}(\zeta)=\ln f_{k}^{\prime}\left(w_{k}(\zeta)\right)+\ln w_{k}^{\prime}(\zeta), \zeta \in D_{n k}^{\prime \prime} .
$$

Let $\gamma_{k, \zeta}^{m}$ be closed rectifiable curves in $D_{n k}^{\prime \prime}$ converging to $\gamma_{k}$ as $m \rightarrow \infty$. Then the curves

$$
\gamma_{k, z}^{m}=\left\{z: z=w_{k}(\zeta), \zeta \in \gamma_{k, \zeta}^{m}\right\}
$$

possess similar properties. By inequalities 4.1 we obtain:

$$
\begin{aligned}
& \int_{\gamma_{k, \zeta}^{m}}\left|\ln f^{\prime}(\zeta) d \zeta\right| \leqslant M_{1} \int_{\gamma_{k, z}^{m}}\left|\ln f_{k}^{\prime}(z) d z\right|+M_{2}, \quad k=\overline{1, n}, \\
& \int_{\gamma_{k, z}^{m}}\left|\ln f_{k}^{\prime}(z) d z\right| \leqslant M_{3} \int_{\gamma_{k, \zeta}^{m}}\left|\ln f^{\prime}(\zeta) d \zeta\right|+M_{4}, \quad k=\overline{1, n},
\end{aligned}
$$

where $M_{l}, l=\overline{1,4}$ are positive constants.

Assume that $\Omega_{n}=f\left(E_{n}\right)$ belongs to the Smirnov class and we represent $\ln \left|f^{\prime}(z)\right|$ by the Green formula. Then in accordance [14] the function $\ln f^{\prime}$ belongs to the class $H_{1}\left(D_{n}\right)$. On the base of the definition of the classes $H_{1}$ and inequality (4.3) we conclude that $\ln f_{k}^{\prime} \in H_{1}\left(D_{n k}\right)$. Therefore, $\Omega_{n k}$ is the domain in the Smirnov class. 
The inverse statement can be proved in the same way, just instead of inequality (4.3), one should employ 4.2 .

By means of Lemma 4.1 , we can easily estimate the growth rate in $r=\left|\zeta-a_{k}\right|, R_{k}-\varepsilon \leqslant r \leqslant$ $R_{k}$ at the boundary $\gamma_{k}$ for many functionals on univalent functions in $D_{n}$ if we know similar estimates for the functions, which are univalent in a circle or in the exterior of a circle. Exactly this is employed in the proof of the following statement.

Lemma 4.2. If $f$ is holomorphic and univalent in $D_{n}$, then

$$
\sup _{z \in D_{n}}\left(\left|\frac{f^{\prime \prime}(z)}{f^{\prime}(z)}\right| \prod_{k=1}^{n}\left(R_{k}-\left|z-a_{k}\right|\right)\right)=M(f)<\infty .
$$

Proof. If $f_{k}$ is univalent in $D_{n k}$, then by the known results (see, for instance, [19], [23]) we can write

$$
\sup _{z \in E_{n k}}\left(\left|\frac{f_{k}^{\prime \prime}(z)}{f_{k}^{\prime}(z)}\left(R_{k}-\left|z-a_{k}\right|\right)\right|\right) \leqslant M_{1}<\infty .
$$

It follows from 4.1 that

$$
\frac{f^{\prime \prime}(\zeta)}{f^{\prime}(\zeta)}=\frac{f_{k}^{\prime \prime}\left(w_{k}(\zeta)\right)}{f_{k}^{\prime}\left(w_{k}(\zeta)\right)} w_{k}^{\prime}(\zeta)+\frac{w_{k}^{\prime \prime}(\zeta)}{w_{k}^{\prime}(\zeta)}, \zeta \in D_{n k}^{\prime \prime} .
$$

By 4.1, 4.4 and the inequality $C_{5} \leqslant\left|w_{k}^{\prime \prime}(\zeta) / w_{k}^{\prime}(\zeta)\right|<C_{6}$ we obtain the relation

$$
\sup _{\zeta \in D_{n k}^{\prime \prime}}\left(\left|\frac{f^{\prime \prime}(\zeta)}{f^{\prime}(\zeta)}\left(R_{k}-\left|\zeta-a_{k}\right|\right)\right|\right) \leqslant M_{2}<\infty
$$

which implies immediately the statement of the lemma.

Now we consider the domain $\Omega_{n}$ not belonging to Smirnov class and bounded by Jordan rectifiable curves. By the Riesz theorem we have $f^{\prime} \in H_{1}\left(D_{n}\right)$ and this is why we can use parametric representation (2.6). It is obvious that we have the following structural formula for the function mapping some canonical domain $D_{n}$ onto $\Omega_{n}$ :

$$
\begin{aligned}
& f(z)=\int z^{\delta} \Theta^{-1}(z) \exp \left\{\sum_{k=1}^{n} \frac{1}{2 \pi} \int_{-\pi}^{\pi} S_{k}\left(z, z_{k}(\theta)\right) d \nu_{k}(\theta)-\alpha\right\} d z, \quad z_{k} \in \gamma_{k}, \\
& \alpha=\sum_{j \neq k} \frac{1}{2 \pi} \int_{-\pi}^{\pi} S_{j}\left(z_{k}, z_{j}(\theta)\right) d \nu_{j}(\theta), \quad k=\overline{1, n} .
\end{aligned}
$$

Here $\nu_{k}$ is a function with a bounded variation and its expansion involves a non-trivial singular component $\mu_{k}(\theta), k=\overline{1, n}$. Let the simply-connected domain $\Omega_{n}$ is bounded by Jordan rectifiable curves $\Gamma_{k}, k=\overline{1, n}$. We interested in the question what restrictions are imposed for mapping function 4.5 by the latter conditions.

Theorem 4.1. If function (4.5) maps $D_{n}$ onto $\Omega_{n}$ in the univalent and conformal way, then there exists a constant $K>0$ such that $\nu_{k} \in \Lambda(K)$ for some $k=1,2, \ldots, n$.

Proof. We represent expression (4.5) in the annulus $D_{n k}^{\prime}$ as (see [17]):

$$
\ln f^{\prime}(z)=\delta \ln z-\ln \Theta(z)+\frac{1}{2 \pi} \int_{-\pi}^{\pi} \frac{z+z_{k}-2 a_{k}}{z-z_{k}} d \nu_{k}(\theta)+\varphi_{k}(z), \quad z \in D_{n k}^{\prime},
$$


where $\varphi_{k}$ is a function holomorphic in the domain $D^{k}=\bigcap_{j \neq k} D_{n j}$ and is hence holomorphic in the annulus $\bar{D}_{n k}^{\prime}$. Let

$$
q_{k}(z)=\frac{1}{2 \pi} \int_{-\pi}^{\pi} \nu_{k}(\theta) \frac{z+z_{k}-2 a_{k}}{z-z_{k}} d \theta
$$

be a function holomorphic in $D_{n k}$. Then on the circumference $\gamma_{k}$ the identity $\operatorname{Re} q_{k}\left(z_{k}(\theta)\right)=$ $\nu_{k}(\theta)$ holds true. By 4.6 we have

$$
i\left(z-a_{k}\right) q_{k}^{\prime}(z)=\ln f^{\prime}(z)-\delta \ln z+\ln \Theta(z)-\varphi_{k}(z), \quad z \in D_{n k}^{\prime} .
$$

Hence, by Lemma 4.2 we find that

$$
\sup _{z \in D_{n k}^{\prime}}\left|\left(R_{k}-\left|z-a_{k}\right|\right) \frac{d}{d z}\left[\left(z-a_{k}\right) q_{k}^{\prime}(z)\right]\right| \leqslant M<\infty .
$$

We map the unit circle $D=\{\zeta:|\zeta|<1\}$ onto the domain $D_{n k}$ by the function $z_{k}(\zeta)=$ $a_{k}+R_{k} / \zeta$. Then we consider a holomorphic in the unit circle function defined by the identity $Q_{k}(\zeta):=q_{k}\left[z_{k}(\zeta)\right]$ and the function $\tilde{Q}_{k}$ defined by the identity $\tilde{Q}_{k}(\theta)=Q_{k}\left(e^{i \theta}\right)$. It is easy to see that

$$
\sup _{\zeta \in D}\left|\left(1-|\zeta|^{2}\right) \frac{d}{d \zeta}\left[\zeta Q_{k}^{\prime}(\zeta)\right]\right|=\sup _{z \in D_{n k}^{\prime}}\left|\left(R_{k}-\left|z-a_{k}\right|\right) \frac{d}{d z}\left[\left(z-a_{k}\right) q_{k}^{\prime}(z)\right]\right| \leqslant M<\infty .
$$

This implies [5] that $\tilde{Q}_{k} \in \Lambda(K)$ with certain constant $K>0$ depending on $M$ only. Establishing a relation between the boundary values of $Q_{k}(\zeta)$ and $q_{k}(z)$, we arrive at the statement of the theorem.

We mention that this theorem extends the known result by Duren, Shapiro and Shields 4 ] for multi-connected domains.

\section{BIBLIOGRAPHY}

1. I.I. Privalov. Boundary properties of analytic functions. 2n ed. Gostekhizdat, Moscow-Leningrad (1950). (in Russian).

2. V.I. Smirnov. Sur la théorie des polynômes orthogonaux à la variable complex // Zhurn. Leningr. Fiz.-Matem. Obsch. 2, 155-179 (1928).

3. M.V. Keldys̆, M.A. Lavrentiev. Sur la représentation conforme des domaines limités par des courbes rectifiables // Ann. Ecole Norm. Ser 3. 54, 1-38 (1937).

4. P.L. Duren, M.S. Shapiro, A.L. Shields. Singular measures and domains not of Smirnov type // Duke Math. J. 33:2, 247-254 (1966).

5. A. Zygmund. Trigonometric series. V. 1,2. Cambridge Univ. Press, Cambidge (1959).

6. L.V. Ahlfors, G. Weill. A uniqueness theorem for Beltrami equations // Proc. Amer. Math. Soc. 13:6, 247-254 (1962).

7. P.L. Shabalin. The univalence of the general solution of an interior inverse boundary value problem // Izv. VUZov. Matem. 12, 92-95 (1975). [Soviet Math. (Izvestiya VUZ. Matematika. 19:12, 77-80 (1975).]

8. F.G. Avkhadiev. Estimates in the Zygmund class and their application to boundary value problems // Dokl. AN SSSR. 307:6, 1289-1292 (1989). [Sov. Math. Dokl. 40:1, 217-220 (1990).]

9. N.G. Makarov. Size of the set of singular points on the boundary of a non-Smirnov domain // Zap. Nauch. Semin. LOMI. 170, 176-183 (1989). [J. Sov. Math. 63:2, 212-216 (1993).]

10. P.W. Jones, S.K. Smirnov. On Smirnov domains // Ann. Acad. Sci. Fennicae Mathem. 24, 105-108 (1999).

11. C. Bishop, L. Carleson, J. Garnett, P.W. Jones. Harmonic measures supported on curves // Pacific J. Math. 138:2, 233-236 (1989). 
12. S. Rohde. On conformal welding and quasicircles // Michigan Math. J. 38:1, 111-116 (1991).

13. V.N. Dubinin. Inequalities for harmonic measures with respect to non-overlapping domains // Izv. Akad. Nauk SSSR. Ser. Matem. 79:5, 47-64 (2015). [Izvestiya: Math. 79:5, 902-918 (2015).]

14. G.Ts. Tumarkin, S.Ya. Havinson. Analytic functions on multiply-connected regions of the class of V. I. Smirnov (class S) // Izv. Akad. Nauk SSSR. Ser. Matem. 22:3, 379-385 (1958). (in Russian).

15. G.Ts. Tumarkin, S.Ya. Havinson. Existence in multiply-connected regions of single-valued analytic functions with a given modulus of boundary values // Izv. Akad. Nauk SSSR. Ser. Mat. 22:4, 543-562 (1958). (in Russian).

16. G.Ts. Tumarkin. Existence condition for analytic majorant of a family of analytic functions // Izv. Arm. Akad. Nauk SSR. Ser. Matem. 17:6, 3-25 (1964). (in Russian).

17. D. Khavinson. Factorization theorems for different classes of analytic functions in multiply connected domains // Pacific J. Math. 108:2, 295-318 (1983).

18. F.G. Avkhadiev. Sufficient conditions for the univalence of quasiconformal mappings // Matem. Zametki. 18:6, 793-802 (1975). [Math. Notes. 18:6, 1063-1067 (1975).]

19. B.G. Osgood. Some properties of $f^{\prime \prime} / f^{\prime}$ and the Poincaré metric // Indiana Univ. Math. J. 31:4, 449-461 (1982).

20. J. Becker, Ch. Pommerenke. Schlichtheitskriterien und Jordangebiete // J. Reine Angew. Math. 354, 74-94 (1984).

21. F.G. Avkhadiev. On injectivity in the domain of open isolated mappings with given boundary properties // Dokl. Akad. Nauk SSSR. 292:4, 780-783 (1987). [Sov. Math. Dokl. 35, 117-120 (1987).]

22. F.G. Avkhadiev. Admissible functionals in injectivity conditions for differentiable mappings of n-dimensional domains // Izv. VUZov. Matem. 4, 3-12 (1989). [Soviet Math. (Izvestiya VUZ. Matematika). 33:4, 1-12 (1989).]

23. F.G. Avkhadiev, K.J. Wirths. Schwarz-Pick type inequalities. Birkhäuser, Basel (2009).

24. G.M. Goluzin. On the parametric representation of functions univalent in a ring // Matem. Sborn. 29(71):2, 469-476 (1951). (in Russian).

Farit Gabidinovich Avkhadiev,

Kazan Federal University,

Kremlevskaya str., 18,

420008, Kazan, Russia

E-mail: avkhadiev47@mail.ru

Pavel Leonidovich Shabalin,

Kazan State University of Architecture and Engineering,

Zelenaya str., 1,

420043, Kazan, Russia

E-mail: pavel.shabalin@mail.ru 